\title{
Superfluidity versus Anderson Localization in a Dilute Bose Gas
}

\author{
T. Paul, ${ }^{1}$ P. Schlagheck, ${ }^{2}$ P. Leboeuf, ${ }^{1}$ and N. Pavloff ${ }^{1}$ \\ ${ }^{1}$ Laboratoire de Physique Théorique et Modèles Statistiques, CNRS, Université Paris Sud, UMR8626, 91405 Orsay Cedex, France \\ ${ }^{2}$ Institut für Theoretische Physik, Universität Regensburg, 93040 Regensburg, Germany
}

(Received 26 February 2007; published 23 May 2007)

\begin{abstract}
We consider the motion of a quasi-one-dimensional beam of Bose-Einstein condensed particles in a disordered region of finite extent. Interaction effects lead to the appearance of two distinct regions of stationary flow. One is subsonic and corresponds to superfluid motion. The other one is supersonic and dissipative and shows Anderson localization. We compute analytically the interaction-dependent localization length. We also explain the disappearance of the supersonic stationary flow for large disordered samples.
\end{abstract}

\section{DOI: 10.1103/PhysRevLett.98.210602}

Interference effects have a strong influence on the transport properties of phase coherent systems. In particular, in one dimension (1D) in the presence of disorder, they lead to Anderson localization (AL), revealed, for instance, by a transmission decreasing exponentially with system size. On the other hand, interactions in a low temperature Bose system may lead to superfluidity (SF), i.e., perfect transmission. Understanding the interplay between these two contrasting tendencies is one of the key issues of the physics of interacting disordered Bose systems. Recent advances in creating and manipulating guided cold atomic vapors and atom lasers (see, e.g., Refs. [1,2]) offer new prospects for studying such transport phenomena in BoseEinstein condensates (BECs). BEC systems are of particular interest in that respect because they are almost perfectly phase coherent, and interaction between their components can be easily modified and modeled. Recently, great experimental efforts have been devoted to the identification of AL in these systems [3-5]. Though not yet observed, the possibility of AL has been pointed out theoretically in the noninteracting regime $[6,7]$, while interactions may play a role in the localization scenario [7].

In this Letter, we study a quasi-1D, weakly interacting $\mathrm{BEC}$, propagating through a disordered potential. In this context, localization has been theoretically studied mainly for effective attractive interactions (see, e.g., [8] and references therein), with less attention on the repulsive case that we consider here (see, however, Refs. [9-11]). In the regime of weak disorder and weak velocity, the condensate depletion and the destruction of SF have been considered theoretically in Ref. [12], while the strong disorder regime has been analyzed numerically in Ref. [13].

Our main result is a new, global picture of coherent transport of an interacting BEC through disorder, with a clear characterization of the different transport regimes where SF and AL can be observed. Our findings are summarized in Fig. 1. The different types of flow are displayed as a function of $v=V / c$ and $L / \xi$, where $V$ is the condensate velocity relative to a disordered potential of spatial extension $L, c$ is the speed of sound, and $\xi$ is the healing length. At low velocities (subsonic regime), the
PACS numbers: 05.60.Gg, 03.75.-b, 05.30.Jp, 42.65.Tg

flow is SF, as expected on the basis of Landau's criterion. The density profile is stationary but locally modified by the external potential at the expense of the creation of a normal fluid fraction, which we compute perturbatively, extending previous results obtained in the limit $v \rightarrow 0$ [12] to finite velocities [Eq. (5)]. In the opposite supersonic regime, a region of stationary flows also exists, but in this case energy dissipation occurs. In this domain, depending on the extent of the disordered potential, the system is either in an Ohmic or in an AL regime (respectively characterized by a transmission decreasing linearly or exponentially with $L$; see below). In between the high and low velocity stationary regimes, there is an intermediate domain, centered around $v \sim 1$, where the flow is time-dependent.

Consider a BEC at rest in a $3 \mathrm{D}$ guide where a disordered potential $U$ of finite spatial extension $L$ is moved in the longitudinal $x$ direction at constant velocity $V>0$. The transverse confinement is such that the "1D mean field regime" [14] holds. The system is then described by an order parameter of the form $\exp \{-i \mu t / \hbar\} \psi(x, t)$, which is

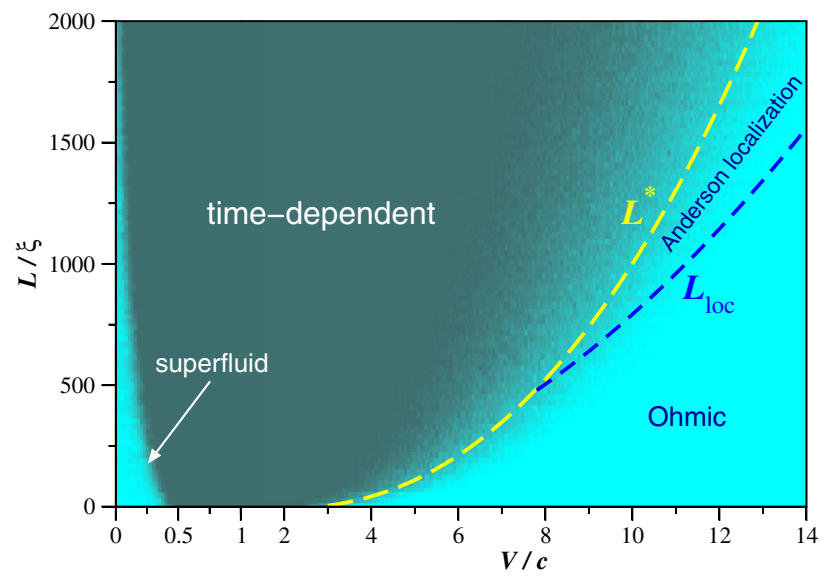

FIG. 1 (color online). Transport of a quasi-1D BEC with normalized velocity $v$ through a disordered potential of length $L$ [Eq. (2), $\lambda=n_{i} \xi=0.5$ ]. Dark region: Time-dependent flow; light blue/gray regions: stationary flow. Note the enlarged scale for $v \in[0,1]$. 
a solution of

$$
i \hbar \frac{\partial \psi}{\partial t}=-\frac{\hbar^{2}}{2 m} \frac{\partial^{2} \psi}{\partial x^{2}}+\left[U(x-V t)+g|\psi|^{2}-\mu\right] \psi .
$$

In the case of particles experiencing an effective repulsive interaction characterized by the 3D $s$-wave scattering length $a>0$, the constant $g$ reads $g=2 h \nu_{\perp} a$, where $\nu_{\perp}$ is the frequency of the transverse harmonic trap [15]. In the stationary regime, where the flow is time-independent in the frame moving with the potential, $\psi$ depends on $x$ and $t$ only through the variable $X=x-V t$. The appropriate boundary condition is $\psi(X \rightarrow-\infty)=\sqrt{n_{0}}$ (where $n_{0}$ is a constant) [16]. The condensate is characterized by a chemical potential $\mu=g n_{0}$, a speed of sound $c=(\mu / m)^{1 / 2}$, and a healing length $\xi=\hbar /(m c)$. We consider a regime where the typical value of the disordered potential is much smaller than $\mu+m V^{2} / 2$. This regime is easily reached experimentally and is important for our purpose because it corresponds to a range of parameters where AL is not blurred by effects connected to "fragmentation of the condensate" (see, e.g., Ref. [2] and references therein).

The picture we draw in the present Letter is generic and does not depend on the specific structure of the disordered potential $U$. We illustrate our findings with a potential of the form

$$
U(x)=\lambda \mu \xi \sum_{n=1}^{N_{i}} \delta\left(x-x_{n}\right),
$$

where the $x_{n}$ are the random, uncorrelated positions of $N_{i}$ delta scatterers uniformly distributed over a length $L$ (with a density $\left.n_{i}=N_{i} / L\right) . \lambda>0$ is the dimensionless strength of a scatterer. This potential has a mean value $\langle U\rangle=$ $\lambda \mu \xi n_{i}$ and a cumulant $\langle U(x) U(0)\rangle-\langle U\rangle^{2}=$ $\left(\hbar^{2} / m\right)^{2} \sigma \delta(x)$, where $\sigma=\lambda^{2} n_{i} \xi^{-2}$. From what is known in the case of the Schrödinger equation, this potential is typical insofar as localization properties are concerned.

Numerically, we solve Eq. (1) using the potential (2) for given $\mu, m, n_{i}, \lambda$, and $V$. We consider 100 realizations of the random potential. The fraction $f$ of potentials for which a stationary solution exists is plotted in Fig. 1 using a gray scale (dark, $f=0$; light blue/gray, $f=1$ ) as a function of the normalized variables $L / \xi$ and $v=V / c$. This normalization rescales all interaction effects. In the following, we characterize the different stationary regimes represented in Fig. 1.

Let us start with the subsonic stationary regime. There, the density profile is easily computed perturbatively to be of the form $n(X)=n_{0}+\delta n(X)$, with [16,17]

$$
\delta n(X) \simeq-\frac{m n_{0}}{\hbar^{2} \kappa} \int_{\mathbb{R}} d y U(y) \exp \{-2 \kappa|X-y|\},
$$

where

$$
\kappa=\frac{m}{\hbar}\left|c^{2}-V^{2}\right|^{1 / 2}
$$

is an effective wave vector. From Eq. (3), $\delta n(X \rightarrow \pm \infty)=$
0 and the density is perturbed only in the region of the potential. This corresponds to perfect transmission of the condensate through the disordered potential. There is no energy dissipation nor drag exerted on the potential, a characteristic feature of SF [18]. We have verified these properties numerically and also checked the stability of the SF flow. There is also a nonsuperfluid fraction, since a momentum $P=\hbar \int d x \delta n \partial_{x} S$ [19] can be associated to the flow (where $S$ is the phase of the order parameter). In the spirit of Landau's approach for determining the normal fraction in liquid ${ }^{4} \mathrm{He}$ (see also [20]), one can associate to this momentum a normal mass $M_{n}=P / V$. In the perturbative limit where Eq. (3) holds, one gets

$$
\frac{M_{n}}{M}=\frac{m^{2}}{2 \hbar^{4} \kappa^{3} L} \int_{\mathbb{R}^{2}} d y_{1} d y_{2} U\left(y_{1}\right) U\left(y_{2}\right) K\left(y_{2}-y_{1}\right),
$$

where $K(y)=(1+2 \kappa|y|) \exp \{-2 \kappa|y|\}$ and $M=m n_{0} L$ is the mass of a region of size $L$ of the unperturbed condensate. The ratio $M_{n} / M$ corresponds to the normal fraction present in the disordered region. For a potential of type (2), at zero velocity, in the scarce impurities limit $\left(1 \gg n_{i} \xi\right)$, Eq. (5) yields $M_{n}(0) / M=\lambda^{2} n_{i} \xi / 2$, in accordance with the findings of Refs. [12,20]. The present approach allows one to extend this result to finite velocities, yielding (for $\left.\kappa \gg n_{i}\right): M_{n}(v) / M_{n}(0)=\left(1-v^{2}\right)^{-3 / 2}$.

The situation changes drastically as the velocity increases. Starting from the SF subsonic region (with fixed $U$ and $L$ ), at some critical velocity the system enters a domain where the flow becomes unsteady (dark region in Fig. 1). The velocity at which this transition occurs depends very much on the specific form of the potential $U$ (see, e.g., the discussion of the influence of the value of $\lambda$ for a regular array of equally spaced impurities in Ref. [21]). If $v$ further increases, one gets into a new stationary region (now supersonic). Within this region, if $v$ is large enough, one reaches a domain (qualified as Ohmic in Fig. 1) where perturbation theory holds, yielding [16]

$$
\delta n(X) \simeq \frac{2 m n_{0}}{\hbar^{2} \kappa} \int_{-\infty}^{X} d y U(y) \sin [2 \kappa(X-y)],
$$

where $\kappa$ is given by Eq. (4). In the region upstream with respect to the disordered potential $(X \rightarrow+\infty)$, Eq. (6) corresponds to an oscillatory wave, i.e., to a nonperfect transmission, indicating the dissipative nature of the flow. Equation (6) allows one to express the disorder averaged transmission coefficient as [10]

$$
\langle T\rangle \simeq 1-\frac{L}{L_{\mathrm{loc}}(\kappa)}, \quad \text { where } \quad L_{\mathrm{loc}}(\kappa)=\frac{\kappa^{2}}{\sigma} .
$$

The probability distribution of $T$ can be shown to be

$$
P(T)=\frac{L_{\mathrm{loc}}}{L} \exp \left\{-(1-T) \frac{L_{\mathrm{loc}}}{L}\right\} .
$$

The validity of Eqs. (7) and (8) is confirmed by comparison with numerical results in Figs. 2(a) and 2(c). These equa- 
tions hold for potentials of the form (2), for Gaussian white noise potentials, as well as for correlated noise, but in the latter case the expression of $L_{\mathrm{loc}}(\kappa)$ is modified. In this regime, the transmission decreases linearly with $L$; this is why we call it Ohmic. Equations (6)-(8) are valid in the perturbative regime $|\delta n| \ll n_{0}$ or, equivalently, $L \ll$ $L_{\mathrm{loc}}(\kappa)$. Starting from the Ohmic regime, keeping fixed $v>1$, and increasing the length $L$ of the disordered potential, one enters the interesting nonperturbative domain $L>L_{\mathrm{loc}}$, where AL (i.e., an exponential suppression of the transmission with disorder length) is expected. In this regime, we will show [cf. Eq. (11) below] that the quantity $L_{\text {loc }}$ appearing in Eq. (7) corresponds indeed to the localization length.

We now derive a nonperturbative treatment of the supersonic stationary flow, valid in the AL regime. We consider a disordered potential of type (2) and look for stationary solutions. Between two impurities ( $x_{n}$ and $x_{n+1}$, say), the random potential is zero, and (1) admits a first integral of the form [16]

$$
\frac{\xi^{2}}{2}\left(\frac{d A}{d X}\right)^{2}+W(A(X))=E_{\mathrm{cl}}^{n}
$$

where $E_{\mathrm{cl}}^{n}$ is a constant, $A=|\psi| / \sqrt{n_{0}}$, and $W(A)=\frac{1}{2} \times$ $\left(A^{2}-1\right)\left(1+v^{2}-A^{2}-v^{2} / A^{2}\right)$. Equation (9) has a Hamiltonian form, expressing energy conservation for a fictitious classical particle with "mass" $\xi^{2}$, "position" $A$, and "time" $X$, evolving in a potential $W$ (whose typical shape is displayed in Fig. 3). The integration of Eq. (9) starts from the left, using the initial position $A(X<0)=1$, which corresponds to $E_{\mathrm{cl}}^{0}=W(1)=0$ (supersonic uniform flow; see [16] and Fig. 3). The behavior of $A$ for $X>$ $L$ depends on the final value of $E_{\mathrm{cl}}^{N_{i}}$. A stationary solution exists only if $A(X>L)$ remains bounded, i.e., if $E_{\mathrm{cl}}^{N_{i}}<$ $W\left(A_{1}\right)$ (where $A_{1}$ corresponds to the local maximum of $W$; see Fig. 3). In this case, the transmission coefficient is [22]

$$
T=\frac{1}{1+\left(2 \kappa^{2} \xi^{2}\right)^{-1} E_{\mathrm{cl}}^{N_{i}}} .
$$

This formula is nonperturbative in the deep supersonic regime $v \gg 1$ (i.e., it is not limited by the condition $|\delta n| \ll n_{0}$ ), and $T$, given by Eq. (10), may become very small [contrarily to Eq. (7), which is valid only for values of the transmission close to unity].

In order to describe the density profile in the disordered region and to determine the value of $E_{\mathrm{cl}}^{N_{i}}$, one can devise a diffusion equation describing the evolution of $E_{\mathrm{cl}}$ under the effect of the kicks caused by each randomly placed impurity (cf. Fig. 3). This allows one-through (10) — to compute the disorder average of the transmission and its probability distribution. For $L \gg L_{\mathrm{loc}}$ and $\kappa \gg n_{i}, \xi^{-1}$, one obtains

$$
\langle\ln T\rangle=-L / L_{\mathrm{loc}}(\kappa),
$$

where $L_{\mathrm{loc}}(\kappa)$ is given by Eq. (7), whereas the probability distribution reads

$$
P(\ln T)=\sqrt{\frac{L_{\mathrm{loc}}}{4 \pi L}} \exp \left\{-\frac{L_{\mathrm{loc}}}{4 L}\left(\frac{L}{L_{\mathrm{loc}}}+\ln T\right)^{2}\right\} .
$$

Excellent agreement of the analytical formulas (11) and (12) with the numerical results is found [cf. Figs. 2(b) and 2(d)]. It is interesting to note that the exponential decrease (11) of the mean transmission and the log normal distribution (12) are characteristic features of AL of linear waves with a localization length $L_{\text {loc }}$ (see, e.g., [23]). Hence, "typical" AL is predicted to occur in supersonic interacting BEC systems. Interaction induces a modification of the wave vector in $L_{\mathrm{loc}}$ : The expression (7) for the localization length coincides with the noninteracting one but computed for an effective interaction-dependent wave vector $\kappa$, instead of $k=m V / \hbar$. The repulsive interaction diminishes the available kinetic energy and therefore reduces the localization length with respect to the noninteracting case (since $\kappa<k$ ).

The present analysis also explains the onset of time dependence observed numerically in the supersonic region at fixed $V$ and increasing $L$ (see Fig. 1). Once the average "classical energy" $\left\langle E_{\mathrm{cl}}^{N_{i}}\right\rangle$ exceeds the value $W\left(A_{1}\right)$ (see
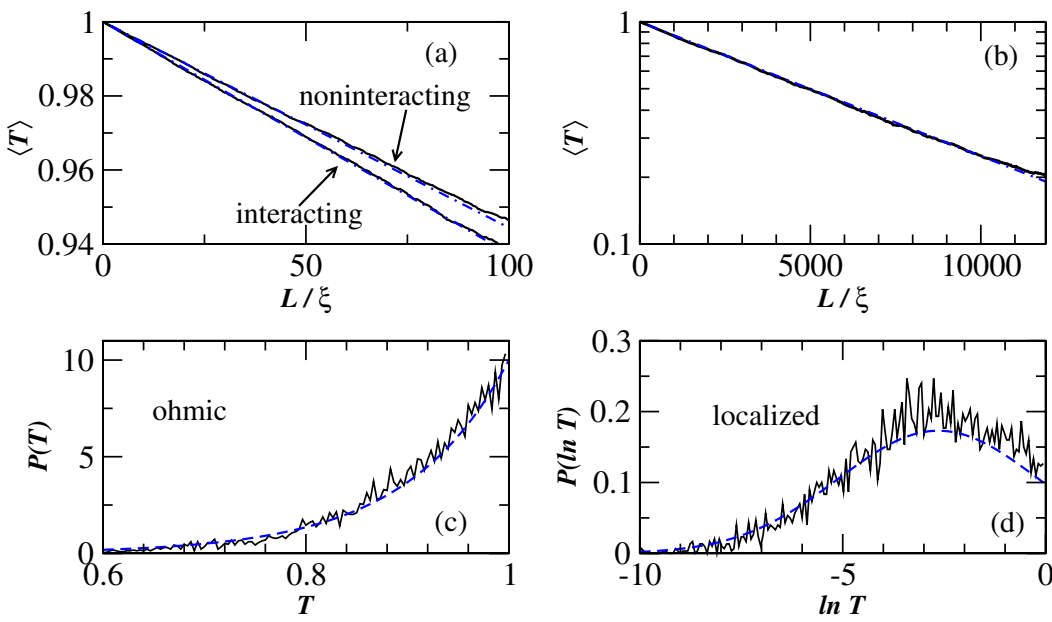

$210602-3$
FIG. 2 (color online). Statistical properties of the transmission $T$ at $v=30$ for potential (2) with $\lambda=n_{i} \xi=0.5$ [except (a), where $v=3$ and $\lambda=0.1]$. Dashed line: Analytical result; solid line: numerics. Top row: Averaged transmission. Analytical and numerical results are almost indistinguishable. The noninteracting results are also represented in (a). Bottom row: Probability distribution of $T$. (c): Ohmic regime, $L / L_{\mathrm{loc}} \simeq 0.1$; (d): AL regime, $L / L_{\mathrm{loc}} \simeq 2.4$. 

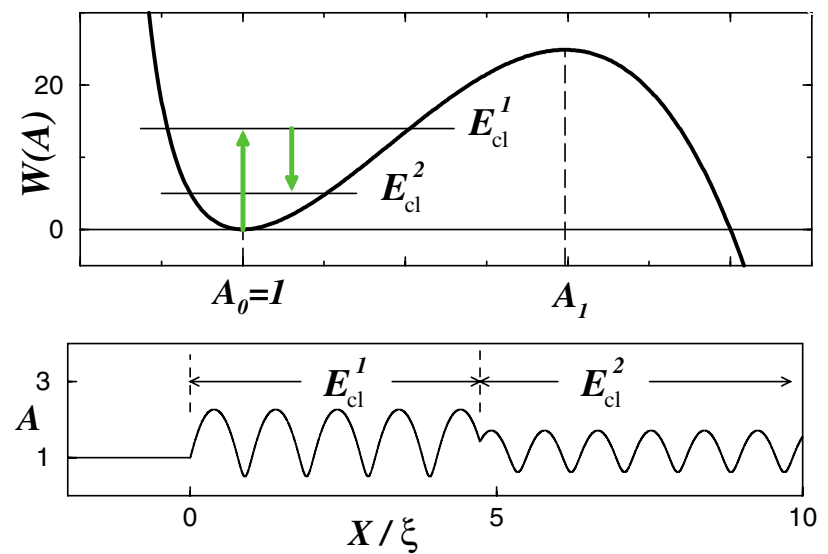

FIG. 3 (color online). Upper panel: $W$ as a function of $A=$ $|\psi| / \sqrt{n_{0}}$ (drawn for $v=4$ ). $A_{0}(=1)$ and $A_{1}$ are the zeros of $d W / d A$. The fictitious particle is initially at rest with $E_{\mathrm{cl}}^{0}=0$. The value of $E_{\mathrm{cl}}$ changes at each impurity. The lower panel displays the corresponding oscillations of $A(X)$, with two impurities (vertical dashed lines) at $x_{1}=0$ and $x_{2}=4.7 \xi$.

Fig. 3), the number of stationary solutions decreases dramatically. This occurs for $L>L^{*}(\kappa)$, with

$$
L^{*}(\kappa)=L_{\mathrm{loc}}(\kappa) \ln \left(v^{2} / 8\right) / 2 .
$$

This expression fits very well the numerically determined frontier of the stationary supersonic domain (see Fig. 1).

At moderate supersonic velocities, Eq. (13) shows that $L^{*}$ is roughly of the same order as $L_{\mathrm{loc}}$, and one cannot reach the regime $L_{\mathrm{loc}} \ll L<L^{*}$, where the log normal distribution (12) is observed. This is the reason why Fig. 2(b) displays the distribution at relatively large velocity $\left(v=30, L^{*} / L_{\mathrm{loc}} \simeq 2.4\right)$. However, the exponential decay (11) of the average transmission is observed for all stationary supersonic velocities, with $L_{\text {loc }}$ given by Eq. (7), as confirmed by Figs. 2(a) and 2(b).

The $v$ dependence of the two relevant length scales $L_{\mathrm{loc}}$ and $L^{*}$ is shown in Fig. 1. Supersonic stationary solutions exist above a critical velocity $v_{1}$ corresponding to $L^{*}\left(\kappa_{1}\right)=0$. One gets $v_{1}=\sqrt{8} \simeq 2.8$, which is the onset of the Ohmic regime. In contrast, AL exists only within the stationary regime for $L_{\text {loc }}<L<L^{*}$ (cf. Fig. 1). This is possible only if $v>v_{2}$, where $L^{*}\left(\kappa_{2}\right)=L_{\text {loc }}\left(\kappa_{2}\right)$, i.e., $v_{2}=e \sqrt{8} \simeq 7.7$. However, for $v>v_{2}$, the difference of $L_{\mathrm{loc}}(\kappa)$ with its noninteracting counterpart $L_{\mathrm{loc}}(k)$ is less than $2 \%$. Hence, interactions play no significant role in the AL regime. They will be relevant, however, in the lowvelocity, small-disorder-length sector of the Ohmic regime. At $v=3$, for instance, they induce a modification of $11 \%$ of the localization length [cf. the comparison of interacting and noninteracting results in Fig. 2(a)].

In summary, we provide a complete picture of the quasi1D transport of a weakly interacting BEC through a disordered potential. We find that interactions have several major and experimentally relevant consequences: (i) the existence of a subsonic SF regime, (ii) the existence of nonsteady flows around $v \simeq 1$, (iii) the renormalization of the localization length in the stationary supersonic regime, and (iv) the introduction of a maximum disorder length scale $L^{*}$ at which AL disappears and time dependence sets in. Explicit methods to characterize experimentally the different regimes through, e.g., the heating rate will be discussed elsewhere.

This work was supported by Grants No. ANR-05-Nano008-02 and No. ANR-NT05-2-42103, by the IFRAF Institute, and by the Alexander von Humboldt Foundation.

[1] A. Greiner et al., arXiv:cond-mat/0701328; W. Guerin et al., Phys. Rev. Lett. 97, 200402 (2006); P. Hommelhoff, W. Hänsel, T. Steinmetz, T. W. Hänsch, and J. Reichel, New J. Phys. 7, 3 (2005); T. Lahaye et al., Phys. Rev. Lett. 93, 093003 (2004); R. Folman et al., Adv. At. Mol. Opt. Phys. 48, 263 (2002).

[2] J. Fortágh and C. Zimmermann, Rev. Mod. Phys. 79, 235 (2007).

[3] J. E. Lye et al., Phys. Rev. Lett. 95, 070401 (2005); C. Fort et al., Phys. Rev. Lett. 95, 170410 (2005).

[4] D. Clément et al., Phys. Rev. Lett. 95, 170409 (2005); D. Clement et al., New J. Phys. 8, 165 (2006).

[5] T. Schulte et al., Phys. Rev. Lett. 95, 170411 (2005).

[6] U. Gavish and Y. Castin, Phys. Rev. Lett. 95, 020401 (2005); R. C. Kuhn et al., Phys. Rev. Lett. 95, 250403 (2005); P. Massignan and Y. Castin, Phys. Rev. A 74, 013616 (2006).

[7] L. Sanchez-Palencia et al., arXiv:cond-mat/0612670; B. Shapiro, arXiv:cond-mat/070134.

[8] S. A. Gredeskul and Y.S. Kivshar, Phys. Rep. 216, 1 (1992).

[9] N. Bilas and N. Pavloff, Phys. Rev. Lett. 95, 130403 (2005).

[10] T. Paul, P. Leboeuf, N. Pavloff, K. Richter, and P. Schlagheck, Phys. Rev. A 72, 063621 (2005).

[11] N. Bilas and N. Pavloff, Eur. Phys. J. D 40, 387 (2006).

[12] K. Huang and H. F. Meng, Phys. Rev. Lett. 69, 644 (1992); S. Giorgini, L. Pitaevskii, and S. Stringari, Phys. Rev. B 49, 12938 (1994).

[13] G.E. Astrakharchik, J. Boronat, J. Casulleras, and S. Giorgini, Phys. Rev. A 66, 023603 (2002).

[14] C. Menotti and S. Stringari, Phys. Rev. A 66, 043610 (2002).

[15] M. Olshanii, Phys. Rev. Lett. 81, 938 (1998).

[16] P. Leboeuf and N. Pavloff, Phys. Rev. A 64, 033602 (2001).

[17] V. Hakim, Phys. Rev. E 55, 2835 (1997).

[18] N. Pavloff, Phys. Rev. A 66, 013610 (2002).

[19] I. V. Barashenkov and E. Yu. Panova, Physica (Amsterdam) 69D, 114 (1993).

[20] G. E. Astrakharchik and L. P. Pitaevskii, Phys. Rev. A 70, 013608 (2004).

[21] D. Taras-Semchuk and J. M.F. Gunn, Phys. Rev. B 60, 13139 (1999).

[22] P. Leboeuf, N. Pavloff, and S. Sinha, Phys. Rev. A 68, 063608 (2003).

[23] B. A. Van Tiggelen, in Diffusive Waves in Complex Media, edited by J.P. Fouque (Kluwer Academic, Dordrecht, 1999), p. 1. 